\title{
Nanotechnology-based inhalation treatments for lung cancer: state of the art
}

This article was published in the following Dove Press journal:

Nanotechnology, Science and Applications

19 November 2015

Number of times this article has been viewed

\author{
Javed Ahmad ${ }^{1, *}$ \\ Sohail Akhter ${ }^{2,3, *}$ \\ Md Rizwanullah' \\ Saima Amin' \\ Mahfoozur Rahman ${ }^{4}$ \\ Mohammad Zaki Ahmad ${ }^{5}$ \\ Moshahid Alam Rizvi ${ }^{6}$ \\ Mohammad A Kamal ${ }^{7}$ \\ Farhan Jalees Ahmad ${ }^{1,2}$ \\ 'Department of Pharmaceutics, \\ ${ }^{2}$ Nanomedicine Research Lab, Faculty \\ of Pharmacy, Jamia Hamdard, New \\ Delhi, India; ${ }^{3}$ Centre de Biophysique \\ Moléculaire(CBM)-CNRS UPR430I, \\ University of Orléans, Orléans \\ Cedex 2, France; ${ }^{4}$ Department of \\ Pharmaceutics, Abhilashi College \\ of Pharmacy, Mandi, HP, India; \\ ${ }^{5}$ Department of Pharmaceutics, \\ College of Pharmacy, Najran \\ University, Saudi Arabia; ${ }^{6}$ Department \\ of Biosciences, Jamia Millia Islamia, \\ New Delhi, India; ${ }^{7}$ Metabolomics and \\ Enzymology Unit, King Fahd Medical \\ Research Center, King Abdulaziz \\ University, Jeddah, Saudi Arabia \\ *These authors contributed equally \\ to this work
}

Correspondence: Sohail Akhter

Centre de Biophysique Moléculaire (CBM)-CNRS/ University of Orléans UPR430 I, Rue Charles Sadron, 4507I

Orléans Cedex 2, France

$\mathrm{Tel}+33699908746$

Email sohailakhtermph@gmail.com

\begin{abstract}
Considering the challenges associated with conventional chemotherapy, targeted and local delivery of chemotherapeutics via nanoparticle (NP) carriers to the lungs is an emerging area of interest. Recent studies and growing clinical application in cancer nanotechnology showed the huge potential of NPs as drug carriers in cancer therapy, including in lung carcinoma for diagnosis, imaging, and theranostics. Researchers have confirmed that nanotechnology-based inhalation chemotherapy is viable and more effective than conventional chemotherapy, with lesser side effects. Recently, many nanocarriers have been investigated, including liposomes, polymeric micelles, polymeric NPs, solid lipid NPs, and inorganic NPs for inhalation treatments of lung cancer. Yet, the toxicity of such nanomaterials to the lungs tissues and further distribution to other organs due to systemic absorption on inhalation delivery is a debatable concern. Here, prospect of NPs-based local lung cancer targeting through inhalation route as well as its associated challenges are discussed.
\end{abstract}

Keywords: nanoparticles, lung cancer, inhalational chemotherapy, drug targeting, nanotoxicity

\section{Introduction}

At present, lung cancer causes $23 \%$ of total cancer-related mortality worldwide, surpassing the combined mortality caused by breast, colon, and prostate cancer. ${ }^{1-4}$ Moreover, it is also recognized as the cancer having most intense fatality, with minimal survival rate. Lung cancer is mainly categorized into two forms; namely non-small-cell lung carcinoma (NSCLC) (major and common form that comprises of $85 \%$ cases) and small-cell lung carcinoma (SCLC). ${ }^{1,2}$ NSCLC is further categorized as epidermoid, large cell, bronchoalveolar, adenocarcinoma, and squamous cell carcinoma. ${ }^{1,2,5-7}$ These NSCLC forms are histologically distinct from each other. It is responsive to the chemotherapy; however, their response differs against a specific therapy. On the other hand, SCLC rarely occurs but shows fast metastasis and aggressive growth, with average survival of merely 4 months if not treated. ${ }^{5}$ Pathophysiology of SCLC indicates that it commences from neuroendocrine tumors, and therefore it contains neurosecretory vesicles and neurofilaments. ${ }^{6}$ While, SCLC is very aggressive in nature, its treatment by radiation and chemotherapy has better responsiveness than NSCLC. ${ }^{1,2,6}$ Irrespective of the different types of lung cancer, the major reason behind the poor survival rate, like most other cases of cancer, is failure of early detection and confirmation only made at the stage IV. ${ }^{8}$ Long-term survival and improved quality of life for lung cancer patients under conventional cancer therapies is still not fulfilled. ${ }^{9}$ Most of the chemotherapeutics are available as intravenous (iv) formulations. Moreover, some important chemotherapeutics used against lung cancers 
are very lipophilic in nature and required higher doses and/or surfactant-based solubilization to improve the systemic drug availability. In addition, oral administration of cancer chemotherapeutics is often limited due to first-pass metabolism..$^{10-12}$ Systemic drug bioavailability is not the only concern here, as even at higher dose or systemic availability, only limited quantity of drugs is delivered to lung tumor. Due to their nontargeting nature, majority of chemotherapeutics act on normal tissues, leading to adverse effects. ${ }^{12}$ Local targeting in lung cancer with the opportunity of minimal systemic exposure can play an important role in safer chemotherapy with better patient compliance. In this direction, drug delivery through inhalation route shows the enormous potential for lung cancer targeting. This offers some obvious advantages such as avoidance of first-pass metabolism, fewer systemic side effects, and a needle-free approach that offer better comfort to the subjects. ${ }^{12-14}$ The progress of pharmaceutical nanotechnology successfully deals with the challenges of drug delivery in cancer chemotherapeutics. Since, nanoparticles (NPs) have extraordinary characteristics like small particle size, large surface area, and the capability of changing their surface properties, they therefore have several advantages over other delivery system. ${ }^{14}$ Additionally, it has been proven that NP-based drug delivery systems assure passive (sizebased targeting due to their size up to $\sim 100 \mathrm{~nm}$ ) as well active targeting (surface functionalization by targeting ligand) and enhanced therapeutic efficacy of anticancer agents. ${ }^{15} \mathrm{NPs}$ can restrict the biodistribution profile and target the drug to the tumor regions, which increases therapeutic efficiency and reduces nonspecific toxicity of anticancer drugs. Moreover, NPs possess good biocompatibility; many of these materials are biodegradable and have the capability to protect nucleic acids from degradation and deliver the biotherapeutic to tumor cells in vivo. ${ }^{16,17}$ While significant work has been done in recent years to target lung cancer using different types of NPs, majority of them were administered iv, which remained with limited success and poor site-specific drug availability in lung cancer. ${ }^{18,19}$

Over the past decade, a new direction in nanotechnology has been raised to focus of targeting to the lungs diseases including cancer. The focus is to combine the nanotechnology-based therapeutic delivery with pulmonary/inhalational route of administration. This strategy has been encouraged due to the possible usefulness of lung as a portal for drug entrance, including peptides and proteins. The lungs are well-organized entrance for drugs to the bloodstream as they have large surface area for absorption ( $100 \mathrm{~m})$, with very thin absorption membrane $(0.1-0.2 \mu \mathrm{m})$. Furthermore, the lungs show comparatively lesser local metabolic activity, and unlike the oral route of drug administration, pulmonary/inhalation route is not vulnerable to first-pass metabolism. ${ }^{19,20}$ Nanocarriers through inhalational route offer many advantages like; 1) they achieve uniform distribution of drug among the alveoli, 2) better solubilization of the drug, 3) sustained drug release which subsequently decreases dosing frequency, 4) better patient compliance, 5) lesser side effects, and 6) improved drug internalization to the cells. ${ }^{21,22}$ Therefore, targeted inhalational NP delivery to the lungs is a potential area of research in cancer nanotechnology that catches the attention of many formulation scientists, oncologists, and biomedical researchers. Here, we discuss the challenges in delivery of chemotherapeutics to lung cancer, the significance of applying inhalational NPs in lungs cancer drug targeting, and the concern of toxicity in using this approach.

\section{Challenges in inhalation nanochemotherapy}

The recent decade has witnessed huge growth in the development of nanocomposite-based drug delivery systems. The current ongoing research is trying to develop safe, efficient, and feasible nano-based drug delivery for highly sensitive imaging action and better therapeutic applications. So far, nanotechnology-based research has been facing numerous challenges in transforming NPs to nanomedicines to clinical practice. During the synthesis of such carrier systems, complications are seen in the robust designing of an appropriate size particle to carry the drug/gene payload efficiently. Nonuniform size distribution, irregular structure/shape, biocompatibility compliance, undefined surface chemistry, and robust reproducibility are the key developmental concerns. Concerning the disease state, delivery of NPs in lung cancer has faced numerous challenges that include improper physiochemical properties of the particles and biological barriers due to lung anatomy and physiology. During clinical stage, nanomedicine-based systems develop striking hindrances such as immune reaction, higher clearance from circulation, and lower efficiency of targeting. Therefore, comprehensive knowledge about biological behavior of nano drug carriers is crucial to achieve the maximum efficient drug delivery. Optimization of physiochemical parameters is an utmost requisite in determining the particle-particle interaction within the biological atmosphere, agglomeration, adsorption of macromolecules onto NP surface, and their intracellular uptake. NPs (size $<200 \mathrm{~nm}$ ) with spherical shape easily move to tumor vasculature, and this process is being exploited to enhance permeation and retention 
(EPR) effect. ${ }^{23}$ Like other cancerous tissues, the occurrence of fine blood vasculature in lungs and poor lymphatic flow facilitates the EPR effect, which enhances the entry of NPs into the tumors. However, NPs with a size less than $50 \mathrm{~nm}$ are less likely to be retained in the tumor tissues for extended period of time. Active targeting involves decorating the NP surface with specific ligands to facilitate the interaction with overexpressed receptor on tumor cells. Various methods are exploited for size optimization of NPs; however, they end up with distorted release of encapsulated drug. Therefore, it is a challenge to keep the size up to $200 \mathrm{~nm}$ in the production cycle. Surface charge plays a key role in determining the fate of NPs in vivo, and further, their particle interaction and agglomeration are mainly dependent upon the zeta potential of the nanocomposites. Cationic polymers like $N$-[1-(2,3-dioleyloxy)propyl]- $N, N, N$-trimethylammonium chloride, dioleoyl phosphatidylethanolamine, etc are sometimes used with engineered NPs for efficient delivery of drug to cancer cells; however, often they cause hemolytic action and raise the issue of safe delivery. ${ }^{24}$ Another concern is instability of nanocarriers due to accumulation of NPs in physiological environment. Once the aggregates are formed, it is quite hard to separate or redisperse the NPs after forced application. It can also cause drug leaching and lower the drug loading and therapeutic efficacy. Nowadays different functional groups are attached to the NP surface for anchoring of different drugs/biomolecules, but they produce complex systems that are often hard to mimic. Considering the effect of drug and/or NPs on respiratory tract, including the effect on the lungs, toxicity is an important concern in its application. Direct application of some chemotherapeutics (for example: irinotecan, gemcitabine, paclitaxel, and its congeners) itself triggered the inflammatory reaction in excess exposure to pulmonary tract. The toxicity related issue is further discussed in detail in "Toxicity concern with inhalational nanomedicines" section. Local delivery of drug or drug-carrying particles to the lungs required inhalation devices such as pressurized metered dose inhalers, nebulizers, and dry powder inhalers. Often, the nano-sized dosage form is delivered as nebulization of colloidal dispersions or solid form using pressurized metered dose inhalers and dry powder inhalers. Physical instability such as agglomeration and poor dispensability remained a challenge. Moreover, chemical instability related to hydrolysis or decomposition of drug and carrier materials is an additional and equally important issue. Exhalation and rapid clearance related poor deposition of majority of the inhaled particles of size up to $1 \mu \mathrm{m}$ in the lung is well established. To get rid of this problem, micron-sized powder carriers containing NPs or dispersible NP agglomerates were considered to improve the lungs deposition of inhalational NPs. Altogether, a fair understanding of aerodynamic properties for pulmonary delivery and respiratory tract anatomy/physiology is required, which requires a collaborative effort from pharmacologists, oncologists, and drug delivery scientists.

\section{Inhalational nanomedicine for lung cancer: recent progress}

Although the inhalational route of drug delivery is one of the primordial administration routes, it has received much attention in the recent decade only. Approval of human insulin powder of recombinant DNA for inhalational delivery, for example, Exubera ${ }^{\circledR}$ (later withdrawn by Pfizer citing the poor acceptance of patients and clinicians) and Afrezza ${ }^{\circledR}$ (manufactured by MannKind Corporation, Danbury, CT, USA and approved by US Food and Drug Administration in 2014), further encourage the researchers to exploit this route, particularly for local lung cancer chemotherapy using NPs as drug carriers. A number of particulate systems ranging in micro- and nanoscale have been investigated for selective delivery of chemotherapeutics to the lungs through the inhalational route. Here, we discuss these particulate systems (keeping in mind the scope of our study, we focus only on nanotechnology-based formulation) from the available researches. The summary of the investigated inhalational NPs carrying chemotherapeutics for local targeting of lung cancer are presented in Table 1.

\section{Polymeric nanocarriers}

\section{Polymeric micelles}

The polymeric micelles represent a potential nanocarrier for efficient delivery of anticancer agents..$^{25,26}$ A polymeric micelle is principally formed when the hydrophobic part of the block copolymer is driven to the interior, which can encapsulate a poorly soluble drug, whereas the hydrophilic portion of the block copolymer faces outward to form the shell. Currently, numerous modified polymeric micelles carrying anticancer agents are in preclinical and clinical phases of development. Gill et $\mathrm{al}^{27}$ produced polyethylene glycol (PEG) ${ }_{5000}$-distearoylphosphatidylethanolamine $\left(\mathrm{PEG}_{5000}-\mathrm{DSPE}\right)$ micelles bearing paclitaxel through solvent evaporation technique. Their observations showed that the $\mathrm{PEG}_{5000}$-DSPE micelles administered intratracheally were capable of maintaining maximum paclitaxel concentrations in lungs for long duration. Intratracheal-delivered polymeric micelles containing paclitaxel exhibited highest aggregation 


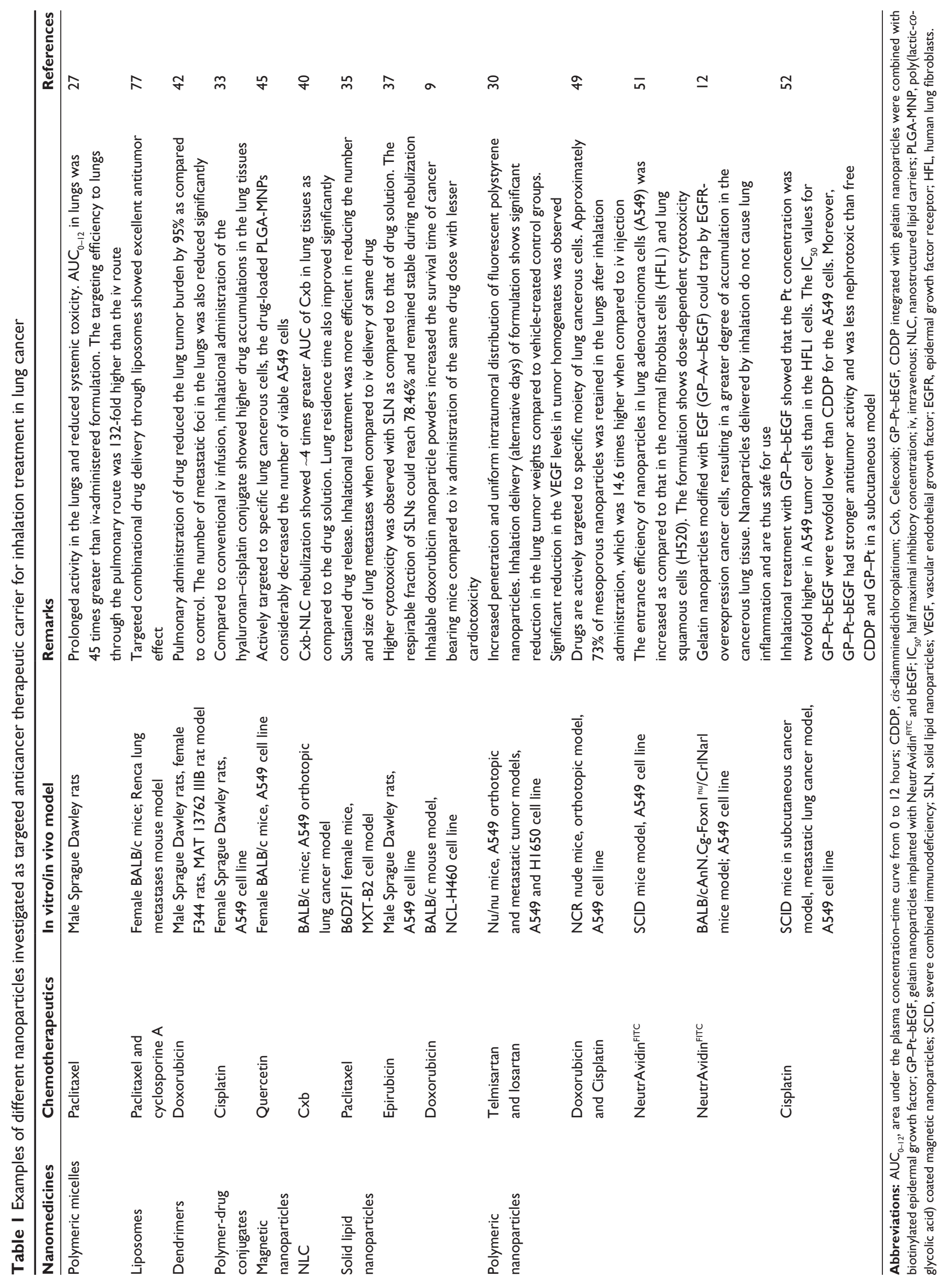


of paclitaxel in the lungs, with $\mathrm{AUC}_{0-12}$ (area under the plasma concentration-time curve from 0 to 12 hours) in lungs 45 times greater than iv-administered formulation and 3 times greater than intratracheally administered taxol. Concentration of paclitaxel in other tissues and plasma was found to be considerably low. ${ }^{27}$ Furthermore, toxicity studies demonstrated that there was no considerable increase in degree of lung injury markers in $\mathrm{PEG}_{5000}-\mathrm{DSPE}$-treated group in comparison to the saline-treated group.

\section{Polymeric nanoparticles}

Polymeric NPs are extensively explored nowadays for their remarkable potential as a drug delivery system for anticancer compounds. They are prepared either by encapsulation, dissolution, and entrapment of drug in biodegradable polymers or by embedding drug in polymeric matrix. Roa et $\mathrm{al}^{9}$ investigated the therapeutic effect of inhaled doxorubicin (DOX)-loaded NPs in a cancer-bearing mouse model (BALB/c model). Spray and freeze drying methods were used to combine DOX-loaded NPs with inhalable effervescent and noneffervescent carrier particles. Cytotoxicity was determined in human NSCLC cell line NCL-H460. Their study suggested that inhalable DOX NP powders enhanced the survival time of cancer-bearing mice with lesser cardiotoxicity in comparison with iv administration of the same dose of drug. Likewise, Kalantarian et $\mathrm{al}^{28}$ designed 5-fluorouracil (5-FU) NPs by supercritical antisolvent process that permits single-step production of 5-FU NPs. The aerodynamic behavior of the NPs was assessed in vitro and demonstrated that the respiratory fraction can increase up to $21 \%$ by the application of the mixture of coarse and fine lactose as a carrier. Furthermore, Tomoda et $\mathrm{al}^{29}$ produced inhalable nanocomposite for the treatment of lung cancer.

They developed the poly(lactic-co-glycolic acid) (PLGA) NPs loaded with cancer chemotherapeutics 6-\{[2-(dimethylamino)ethyl]amino $3-3$-hydroxyl-7H-indeno[2,1-c] quinolin-7-one dihydrochloride in the form of nanocomposite ( size $200 \mathrm{~nm}$ ) to effectively deposit the particles deep within the lung tissue. It was observed that the cytotoxicity of nanocomposite particles against A549 cells was greater than that of free drug. When the nanocomposite particles were administered in rats by inhalation, drug concentration in the lung was much higher than that in plasma. Furthermore, drug concentration in lungs administered by inhalation of NPs was much higher than that after iv administration of free drug. In another study, Godugu et $\mathrm{al}^{30}$ developed NPs for inhalational delivery in lung cancer. The rationale behind their study was to assess the effect of telmisartan
(Tel) and losartan (Los) on intratumoral distribution of NPs and anticancer effects in lung cancer. A549 and H1650 NSCLC cells were used to perform in vitro cytotoxic studies by delivering the drugs orthotopically and metastatically to $\mathrm{Nu} /$ nu mice. They observed that Tel and Los exhibited significant anticancer effects in orthotopic and metastatic lung tumor models. Inhalational delivery of Tel showed enhanced antifibrotic effect as compared to Los. Tel, by virtue of its dual pharmacophoric nature, could be an ideal candidate for combination therapy to improve the NP's intratumoral distribution and anticancer effects. Tel-induced antifibrotic effects can be used to improve the efficiency of nanotherapeutics in different types of tumors. Tel, when administered inhalationally at a minimum dose of $1.12 \mathrm{mg} / \mathrm{kg}$, showed significant fibrolysis and increased intratumoral distribution of NPs in lung tumors. ${ }^{30}$ Poor lung deposition and fast exhalation is a major limitation against lung targeting by inhalational NPs. Recently, attempts have been made to overcome this issue and make use of NPs effectively in targeting lung cancer. ${ }^{31}$ For example, the most common strategies are using a special aerosolizer that can generate particles as micron-sized liquid droplets, applying nanocomposite microparticles and NPs of size cutoff more than $500 \mathrm{~nm}$. Choi et $\mathrm{al}^{31}$ showed the application of special aerosolizer for better lung deposition of NPs. Most recently, the well-defined lung pharmacokinetics and antitumor efficacy in BALB/c nu/nu mice bearing $\mathrm{H} 226$ cell-induced metastatic tumors was ascribed for inhalable TRAIL (apoptosis-inducing ligand) adsorbed NPs of albumin (human serum albumin) coupled with DOX. ${ }^{31}$ It was found that this inhalable NP showed synergistic cell killing and significantly improved the lung deposition at its center and spread throughout. It remained in the lungs for and gradually released the DOX over 3 days. ${ }^{31}$ In another study by Azarmi et al, ${ }^{32}$ they developed DOX-loaded NPs were incorporated as a colloidal drug delivery system into inhalable carrier particles by a spray-freeze-drying method. The cytotoxic effects of free DOX, blank NPs, or DOX-loaded NPs were evaluated on H460 and A549 lung cancer cells. They observed that the drug-loaded NPs carried as dry powders demonstrated a concentration-related increase in in vitro cytotoxicity. This study encourages the strategy of applying NPs in the local treatment of lung cancer as a drug delivery vector.

\section{Polymer-drug conjugates}

The hydrophilic polymers conjugated to proteins and anticancer drugs are one of the most extensively explored approaches for the drug delivery, which establishes polymer therapeutics 
as one of the first classes of anticancer nanomedicine. The prospects of using more sophisticated polymer-based vectors in chemotherapeutics are expanding day by day. Xie et $\mathrm{al}^{33}$ produced inhalational cisplatin-hyaluronan (HA-Pt) combination to treat lung cancer and studied the comparison with conventional cisplatin (cis-diamminedichloroplatinum or CDDP) iv infusion. Cytotoxicity studies were carried out in human lung cancer cell line A549. Their results showed that as compared to conventional CDDP iv infusion, the HA-Pt lung instillation group had not only higher platinum accumulations in the lung tissues and the draining lung surrounding nodes, but also demonstrated a sustained release plasma profile with a reduced peak plasma concentration.

\section{Lipidic nanocarriers}

\section{Solid lipid nanoparticles}

Recently, solid lipid nanoparticles (SLNs) have gained huge notice for delivery of drugs, particularly poorly water soluble drug candidates. They offer improved properties by combining the benefits of liposomes, NPs, and nanoemulsions. They are usually prepared by high-pressure homogenization or microemulsification technique, where drug is efficiently entrapped in a lipid matrix. ${ }^{34}$ SLNs overcome the general limitations of polymeric systems by exhibiting low toxicity due to the presence of biodegradable lipid, and an extremely small size which facilitates circumventing of reticuloendothelial system (RES). Videira et $\mathrm{al}^{35}$ carried out the preclinical evaluation of a pulmonary delivered paclitaxel-loaded lipid nanocarrier for studying antitumor effect in lung cancer. They used MXT-B2 cell lines for cytotoxicity assay. It was found that inhalational treatment of paclitaxel-loaded SLNs was more efficient in reducing the number and size of lung metastases in comparison to iv delivery of same drug. Furthermore, Videira et $\mathrm{al}^{36}$ in another study used glyceryl behenate to produce NPs by melt homogenization method, and it was radiolabelled with ${ }^{99 \mathrm{~m}} \mathrm{Tc}$ using the lipophilic chelator D,L-hexamethylpropyleneamine oxime. Their results revealed an important role of the lymphatic pathway in the uptake of inhaled nanoparticulates. Their study suggested the possibility of pulmonary administration of radiolabelled SLN as a lymphoscintigraphic agent and direct administration of cytotoxic drugs to target lung cancer that may metastasize via lymphatic drainage. Similarly, $\mathrm{Hu}$ et $\mathrm{al}^{37}$ developed an inhalational formulation of epirubicin (EPI)-loaded SLNs (EPI-SLNs) as an inhalable nanomedicine for lung cancer therapy. Cytotoxicity study was carried out in A549 alveolar epithelial cells, and blank SLNs were found to be nontoxic, while higher cytotoxicity of EPI-SLNs was observed in comparison to that of EPI solution. In vitro deposition assessment suggested that SLNs remained stable during nebulization with better respirable fraction in comparison to EPI solutions. In vivo pharmacokinetic analysis demonstrated that the drug concentration attained by inhalation of EPISLNs was found to be greater than the drug concentration in plasma. Furthermore, the drug concentration in lungs after inhalation of EPI-SLNs was much higher than that after administration of EPI solution.

\section{Nanostructured lipid carriers}

Nanostructured lipid carriers (NLCs) have gained expanding scientific and commercial attention in the last few years as alternate carriers for pharmaceutical delivery, particularly cancer chemotherapeutics. NLCs provide the opportunity to overcome the limitations associated with cancer chemotherapeutics, like poor solubility, specificity and steadiness, normal tissue toxicity, as well as targeting of drug against drug-resistant cases. Recently, NLCs of 9-bromo-noscapine (9-Br-Nos-NLCs) (9-Br-Nos; known to have alteration activity of microtubulin polymerization), a poorly water soluble molecule, was developed as a prospect for NPs-based inhalation treatments against NSCLC. ${ }^{38}$ The work ascribed the detailed study on cytotoxicity in lung cancer cells (A549), lung pharmacokinetics, and biodistribution. For better aerodynamic/lungs deposition, 9-Br-Nos-NLCs was mixed with spray dried lactose. ${ }^{38}$ The nanocarrier illustrated enhanced cytotoxicity, apoptosis, and cellular uptake of 9-Br-Nos. Active endocytosis and passive diffusion are considered as main mechanism for improved cellular uptake. ${ }^{38}$ It has been found that the drug exposure was improved with significant enhancement in half-life of the drug in lungs $\left(\sim 3.75\right.$ times higher $T_{1 / 2}$ than 9-Br-Nos powder) after inhalational application. ${ }^{38}$ Taratula et al, ${ }^{39}$ in their investigation, synthesized multifunctional NLCs and evaluated them for effective delivery of an anticancer drug and siRNA straight into the lungs by inhalation. It demonstrated an increase in antitumor activity compared to iv treatment. It has been found that NLCs accumulated predominately in the lungs and, more specifically, in lung cancer cells, leaving healthy organs and nontumorous lung cells intact. It was also observed that it increases the antitumor effectiveness as compared to free drug or similar systems delivered iv. Similarly, Patlolla et $\mathrm{al}^{40}$ designed inhalable celecoxib-encapsulated NLC (Cxb-NLC) to treat lung cancer. It was found that Cxb-NLC exhibited dose- and time-dependent cytotoxicity against A549 cells. Nebulization of Cxb-NLC demonstrated a 4-fold 
higher $\mathrm{AUC}_{\mathrm{t} / \mathrm{D}}$ in lung tissues compared to the Cxb-Soln in BALB/c mice. ${ }^{40}$ The formulation was capable of releasing the $\mathrm{Cxb}$ in controlled manner for extended duration of time, and the aerodynamic diameter was found to be within the nebulization limits. Aerosolization of Cxb-NLC improved the $\mathrm{Cxb}$ pulmonary bioavailability in comparison to solution, and it may perhaps be a good nanomedicine for lung cancer having better patient compliance with minimal dosing intervals.

\section{Dendrimers}

Dendrimers represent a nanodrug carrier that link molecular chemistry to polymer science. ${ }^{41}$ They have different structures depicted by a core, an inner dendritic constitution of extremely branched polymers, and an exterior of multivalent functional groups. The functional group on their surface gets incorporated with charged polar compounds by electrostatic interaction, whereas the uncharged, nonpolar compounds get embedded in the hydrophobic interior. Since they possess both hydrophobic and hydrophilic parts in their structures, different types of drug molecules could be productively entrapped within dendrimers based on their solubility. Kaminskas et $\mathrm{al}^{42}$ produced DOX-conjugated dendrimer for pulmonary administration for the treatment of lung cancer. They explored the utility of a $56 \mathrm{kDa}$ PEGylated polylysine dendrimer, conjugated to DOX, to promote the controlled and prolonged exposure of cytotoxic drug (DOX) to the lung cancer. The anticancer efficacy of inhaled DOX-conjugated dendrimer (D-DOX) for lung cancer was evaluated in the syngeneic MAT 13762 IIIB rat model of lung metastatic cancer. Intratracheal delivery of the D-DOX twice a week led to a more than $95 \%$ decrease in lung tumor burden after 2 weeks, while iv delivery of DOX solution decreased lung tumor burden by only $30 \%-50 \%{ }^{42}$

\section{Inorganic nanocarriers Magnetic nanoparticles}

The magnetic NPs (MNPs) are prepared by either entrapping drug into magnetic micro/nanosphere or embedding as a magnetically-active disc. In magnetic targeting, the liberation of drug in blood circulation is controlled by applying strong magnetic field. Different magnetic materials with a variety of magnetic properties are available, such as magnetite, iron, nickel, cobalt, neodymium-iron-boron, and samarium-cobalt. Furthermore, some liquids can also be strongly magnetized in magnetic field known as ferrofluids. Ferrofluids are principally colloidal suspensions of nanodimension ferromagnetic particles. Currently, commonly used
MNPs are made of iron oxide due to its biodegradability, biocompatibility, superparamagnetic effects, and capability to act as a contrast agent in magnetic resonance imaging. These NPs on dissociation convert into ferritin and/or hemosiderin following their internalization within the lysosomes of RES cells. ${ }^{43}$ As theranostics in lung cancer therapy. $\left(\mathrm{Fe}_{3} \mathrm{O}_{4}\right) \mathrm{NPs}$ used for the localized hyperthermia induced cancer cells, killing, diagnosis, and targeted delivery of chemotherapeutics to the cancer region under the influence of magnetic field. ${ }^{44}$ Verma et $\mathrm{a}^{45}$ produced magnetic core-shell NPs by surface coating of $\mathrm{Fe}_{3} \mathrm{O}_{4}$ MNPs, with a polymer PLGA intended for nebulized drug delivery for the treatment of lung cancer. The polymeric shell of these framed NPs contains a potential anticancer drug quercetin. A549 lung carcinoma cells were utilized for cytotoxicity study. They observed that the mean particle size of the developed MNPs and PLGA-MNPs was 9.6 and $53.2 \mathrm{~nm}$, respectively. There was no cytotoxicity found when $\sim 100 \mu \mathrm{g} / \mathrm{mL}$ PLGA-MNP was administered to the cultured human lung epithelial cells. Therefore, the MNP-based nanocarrier system was considered as biocompatible. Moreover, the drug-loaded PLGA-MNPs considerably decreased the number of viable A549 cells.

\section{Targeting inhalational nanomedicines to the lung cancer}

The pulmonary delivery of nanomedicines possesses the potential to enhance and maintain local drug concentration to treat lung cancer. If the site of action is targeted, the required dose is decreased, which eventually decreases the systemic side effects. Furthermore, variability in oral dosing due to alteration in gastric conditions can be avoided. Generally, it carries several forms of molecular recognition, which facilitates a drug or drug formulation to interact specifically with a disease-causing molecular phenomenon, and/or notably recognizes and binds to target tissues. It targets receptors by utilizing target-specific natural or artificial receptor ligands or target-specific antibodies attached with drugs or drug formulations. One of the examples of targeted drugs is tumor-specific antibody Herceptin ${ }^{\circledR}$ (a clinically approved monoclonal antibody manufactured by Genentech, Inc., San Francisco, CA, USA), which is used for the treatment of breast cancer. Passive targeting refers to the preferred accumulation of drug or drug formulation in a specific tissue depending on its biophysical properties. For instance, the differential accumulation of particles or aerosol droplets in various lung regions is based on their sizes, ie, EPR effect. Drugs can be formulated as solutes or particles in aerosol droplets of appropriate size and used in 
drug delivery. ${ }^{46}$ The term active targeting is frequently used in place of molecular or biological targeting; however, it can precisely be defined as any active process applied on a formulation which will cause localized drug effect. ${ }^{47}$ Thus, it also includes methods of physical targeting where physical force is applied on drug formulations and/or target cells to mediate localized delivery. Examples include electroporation, sonoporation, and magnetic targeting. Aerosol delivery of drugs by inhalation is the easiest way to target cancerous tissues of lung. ${ }^{48}$ Taratula et a ${ }^{49}$ produced mesoporous silica nanoparticles-based drug delivery system to treat lung cancer by inhalation method. This system was effective in delivering anticancer drugs in cancer cells. Anticancer drugs were conjugated with two kinds of siRNA targeted to MRP1 and BCL2 mRNA for inhibition of pump and nonpump cellular resistance in NSCLC, respectively. A549 human lung adenocarcinoma epithelial cells (A549 cell line) were used for in vivo body distribution study. They observed that this system was capable of delivering various anticancer drugs into lung cancer cells in conjugation with siRNA for concurrent initiation of cell death and inhibition of pump and nonpump cellular drug resistance. The drugs and siRNA transported by the targeted mesoporous silica nanoparticlebased drug delivery system preserve their specific activity. Moreover, simultaneous induction of cell death by anticancer drugs and suppression of pump and nonpump resistance by siRNA substantially enhances the drugs' cytotoxicity. Similarly, Okamoto et a $\mathrm{l}^{50}$ produced chitosan-interferon- $\beta$ gene complex powder to treat lung metastasis in mice by inhalation method. Chitosan was used as a nonviral carrier and mannitol, as a dry powder carrier. CT26 cells were used as the cell line for their study. The expression of a luciferase expression plasmid driven by the cytomegalovirus promoter (pCMV-Luc) and plasmid DNA, encoding farnesylated enhanced green fluorescent protein (pEGFP-F), suggested that the genes expressed in both normal and tumorous tissues and the intratracheal powder resulted in higher expression than the iv or intratracheal solution. The average survival time was prolonged by the intratracheal powder of pCMVmurine interferon- $\beta(\mathrm{Mu} \beta)$ administered at a dose of $1 \mu \mathrm{g}$ compared to the untreated control. Furthermore, Tseng et a ${ }^{51}$ produced inhalational gelatin NPs by combination of biotinylated epithelial growth factor (bEGF) for lung cancer treatment. Gelatin nanoparticles (GPs) were used as native carriers, which were implanted with NeutrAvidin ${ }^{\text {FITC }}$ on the particle's surface (GP-Av). Subsequently, the bEGF molecules were combined with NeutrAvidin ${ }^{\mathrm{FTCC}}$, configuring a core-shell-like structure (GP-Av-bEGF). Since A549 have large quantities of EGF receptor, it was therefore found in in vitro cell culture test that GP-Av-bEGF showed greater entrance in adenocarcinoma cells (A549) than that in normal lung cells (HFL1) and lung squamous cells (H520). GPAv-bEGF NPs were administered by inhalation to the lung of SCID (severe combined immunodeficiency) mice and showed that cancerous lung had greater fluoresced agglomeration. This study demonstrated that uptake of GPAv-bEGF by A549 cells is time and dose dependent. In his another study, Tseng et al ${ }^{12}$ modified GPs with NeutrAvidin $^{\mathrm{FITC}}$-bEGF and designed EGF receptor-seeking NPs (GP$A v-b E G F)$. Aerosol droplets of the GP-Av-bEGF were administered by nebulizer to a mouse model (BALB/cAnN. Cg-Foxn $1^{1 \mathrm{nu}} / \mathrm{CrlNarl}$ ) for lung cancer. The cell line used was A549. They observed that the GP-Av-bEGF was effectively bound to the EGFR-overexpressing cells in the lung cancer mouse model. Furthermore, aerosol droplets having appropriate mass median aerodynamic diameter produced by these NPs were accumulated in the lower respiratory tract, and jet nebulization does not alter the targeting functions of EGF. It was found that the GP-Av-bEGF was mainly deposited in the cancerous lung tissue. They also demonstrated that the GPs delivered by inhalation do not cause lung inflammation and are thus safe for use. Thus, it may prove to be a valuable drug carrier when delivered by simple aerosol method to treat lung cancer. Tseng et $\mathrm{al}^{52}$ further developed biotinylated-EGF-modified gelatin NPs carrier to enhance cisplatin accumulation in cancerous lungs through inhalation. They used GPs as carriers of cisplatin (CDDP), with anticipated improved therapeutic effect and reduced side effects. CDDP integrated with GPs (GP-Pt) were combined with bEGF to produce GP-Pt-bEGF having anticancer activity. An in vitro anticancer study showed that the GP$\mathrm{Pt}-\mathrm{bEGF}$ was more effective than free CDDP or GP-Pt, owing to its quick onset of action on the cell cycle and a lower half maximal inhibitory concentration $\left(\mathrm{IC}_{50}\right)$ for the inhibition of A549 cell growth. The in vivo anticancer study demonstrated that GP-Pt-bEGF had better antitumor activity and less toxicity as compared to free CDDP and GP-Pt in a subcutaneous model. Inhalational formulations of GP-PtbEGF administered to mice with lung cancer through aerosol delivery showed that it can target the EGFR-overexpressing cells to get high dosage in the cancerous lungs and decreases the nephrotoxicity of cisplatin. The novel approach of nanomagnetic drug targeting is made feasible with aerosols that composed of magnetically responsive NPs along with 
anticancer drug to specific regions of the lung. This strategy is quite encouraging in accumulating effective doses of drugs in cancerous sites of lungs and thus decreasing the toxic effects. Dames et $\mathrm{al}^{53}$ called their compositions as "Nanomagnetosols", for magnetic drug targeting through the airways. These may be easily produced with state-of-the-art nebulizers which are used clinically and have an appropriate amount of iron oxide NPs. These make them susceptible to magnetic field control. This newer strategy is interesting as it overcomes the normal accumulation process of inhaled aerosol droplets in the lungs, which only permits targeting of the central airways or lung periphery but not local regions of the lungs. Sadhukha et al, ${ }^{54}$ in their investigation, produced MNPs for targeted hyperthermia in lung cancer via inhalation therapy. They assessed the efficiency of tumor-targeted super paramagnetic iron oxide NPs (SPIONs) for magnetic hyperthermia in lung cancer. EGFR-targeted, inhalable SPIONs were synthesized and characterized for targeting lung tumor cells as well as for magnetic hyperthermiamediated antitumor efficacy in a mouse orthotopic model of NSCLC. For this study, cell lines used were A549 and A549-luc (luciferase-transfected A549). Their results show that EGFR targeting enhances tumor retention of SPIONs. Moreover, it was found that the treatment of hyperthermia by targeted SPIONs causes considerable inhibition in lung tumor growth in vivo. Their work demonstrated the potential for developing an effective anticancer treatment modality for the treatment of NSCLC based on targeted magnetic hyperthermia. Similarly, Shim et $\mathrm{al}^{55}$ produced ethylphosphocholine-based nanolipoplexes for inhalational administration of anticancer siRNA for the treatment of lung cancer. Cationic dioleoyl-sn-glycero-3-ethylphosphocholine and cholesterol (ECL)-based nanoliposomes illustrated the maximum pulmonary cellular delivery in vivo and minimum cytotoxicity in vitro. The delivery efficacy of fluorescent siRNA in ECL nanoliposomes was 26.2 times greater than that of naked siRNA in vivo. When treated with Mcll (myeloid cell leukemia sequence 1)-specific siRNA (siMcl1) by means of ECL nanolipoplexes, it decreased the target expression in B16F10 cell lines, while the control luciferasespecific siGL2 in ECL nanolipoplexes did not. It was found that intratracheal delivery of siMcl1 in ECL nanolipoplexes silenced Mcl1 mRNA and protein levels in lung cells in metastatic lung cancer mouse models induced by B16F10 or Lewis lung carcinoma cells. Furthermore, reduced formation of melanoma tumor nodules was observed in the lung.

\section{Toxicity concern with inhalational nanomedicines}

In view of the particulate drug carriers (nano or micro sized) as inhaled dispersed system, local response as increased macrophages usually happen due to the deposition of poor or insoluble nature of the carrier. It is often considered as a nonalarming physiological response. However, prolonged exposure of such particles enhances the deposition (which is an additive if the carriers are nonbiodegradable) that may lead to further intense inflammatory responses such as irritation, cellular injury, edema, phagocytosis impairment, and breakdown in defense mechanisms. Oxidative stress and inflammation-mediated functional disturbances are major toxicity caused by inhalational NPs. Different in vivo studies demonstrated inflammatory effects of NPs as temporary reaction and evaluated whether the degree of the inflammatory reaction is linked to the exposure dose of NPs or not. Actually, the inflammation in lungs was induced by inhalation ${ }^{56,57}$ or instillation exposures ${ }^{58-60}$ of different kinds of nanomaterials. These investigations exposed local conquest of leukocytes, enhanced numbers of inflammatory cells in bronchoalveolar lavage fluid, liberated of LDH, and enhanced cytokine production. Furthermore, characteristic granulomatous responses were observed following pulmonary exposures of carbon nanotubes. ${ }^{61-63}$ Inhalation NPs accumulate in all parts of the respiratory tract, although bigger particles might be extracted out in the upper airways, while smaller ones spread to the distal airways. ${ }^{64-66}$ Considerable quantities of specific particle size ranges can accumulate in each area, for instance, $90 \%$ of NPs of $1 \mathrm{~nm}$ diameter accumulate in the nasopharyngeal area, while only $10 \%$ of these NPs accumulate in the tracheobronchial area, and nearly nothing reaches the alveolar area. On the contrary, 15\% of NPs of $20 \mathrm{~nm}$ diameter accumulate in the nasopharyngeal area, 15\% in the tracheobronchial area, and approximately $50 \%$ in the alveolar area. ${ }^{66}$ The deposition pattern of the particulate matter in lung is affected by disease state. Any kind of particles, including NPs, deposited significantly more in the asthmatic lungs compared to the normal one. Higher deposition may trigger local inflammation and synergistically intensify the preexisting inflammation of disease in case of asthma and chronic obstructive pulmonary disease. In addition to these, irrespective of biocompatibility, the insoluble nondegradable/ slow degradable material-based particles may lead to pulmonary inflammation to some extent on alveoli deposition. It is now established that the use of chemicals which are endogenous to pulmonary area (such as DPPC) in the formulation 


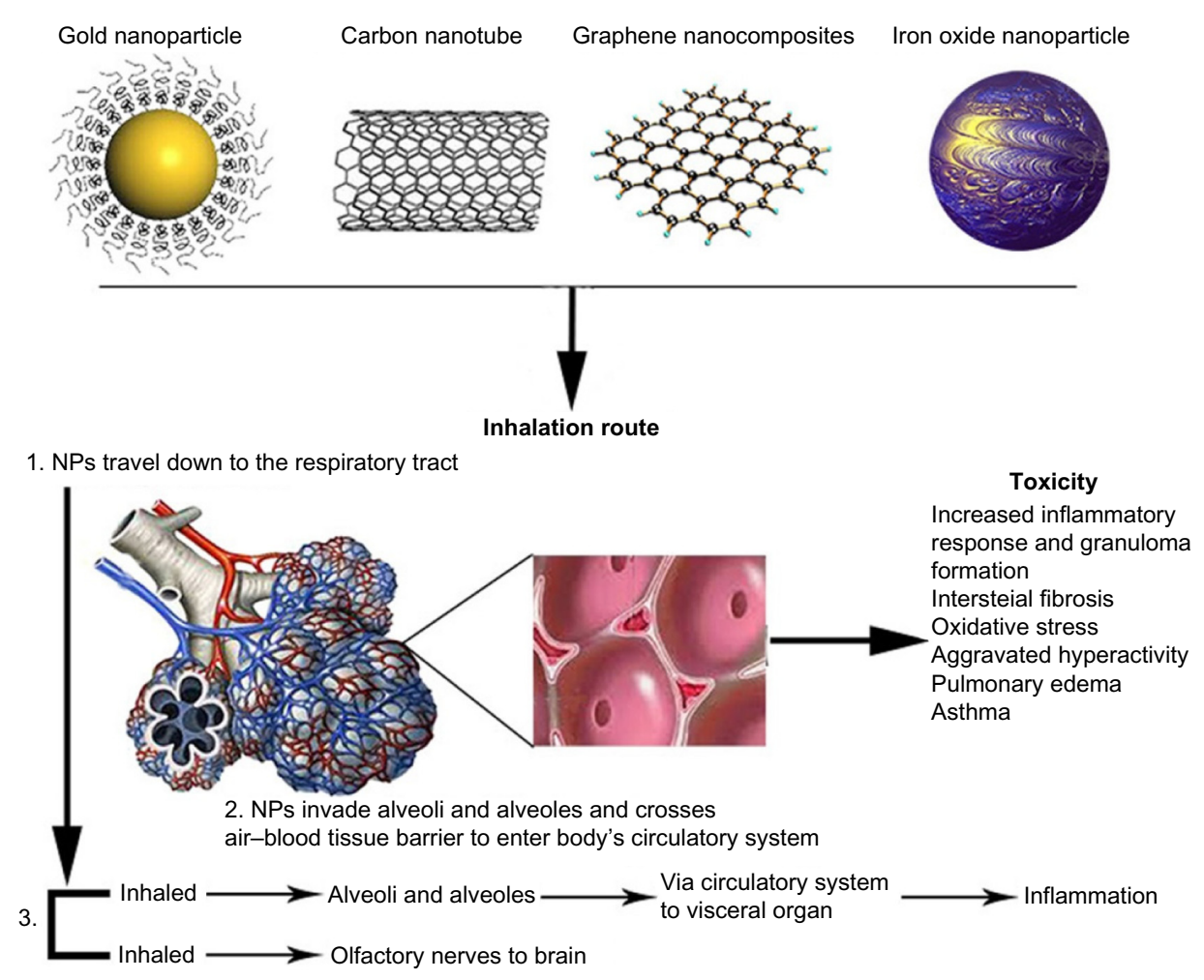

Figure I Organs exposure and toxicity associated with inhalational NPs.

Note: The figure indicates that such NPs can accumulate into the lungs for a very long time and may also enter into the systemic circulation and subsequently reach the other vital organs through blood flow.

Abbreviation: NP, nanoparticle.

significantly reduced the toxicities. Migration of inhaled NPs to the systemic circulation and diffusion to other organs is an important concern of the toxicity (exemplified in Figure 1). Once the NPs are absorbed through the lung epithelium, they can enter the blood and lymph and spread to the cells in the bone marrow, lymph nodes, spleen, heart, and to the central nervous system and ganglia after translocation (Figure 1). ${ }^{65-69}$ Ultrafine particles are labeled as more toxic compared to larger particles with similar chemical composition, owing to their huge surface area, which causes cytotoxicity, allergic reaction, or inflammation. ${ }^{67,70-72}$ Apart from epidemiological and controlled clinical studies, the outcomes of NPs in the respiratory tract are also investigated through inhalation in rodents and in vitro cell culture systems. ${ }^{67}$ Ultrafine particles cause gentle pulmonary inflammatory reactions in rodents and have consequences on extrapulmonary organs. ${ }^{64,67,70}$ Shape and structure of NPs can also influence inhalational toxicity. ${ }^{67,70,73}$ For instance, carbon nanotubes have different pulmonary effects than carbon black and graphite that are bigger structures of same chemical composition. ${ }^{74,75}$ There are fewer epidemiological studies on human exposures and therefore lung cancer threat cannot directly be linked with engineered nanomaterials. The methods causing NP carcinogenicity are unidentified and may include primary genotoxic affront or secondary genotoxic reaction owing to particle-induced inflammation. ${ }^{76}$ This discussion enlightens the need of extensive studies in the nanotoxicity to the lungs to identify the optimal particle characteristics complimenting the pulmonary tract, systemic/nonpulmonary biodistribution and its effect on vital organs, robust technique to analysis, and quantifying the particles concentration in the pulmonary compartments.

\section{Conclusion}

Chemotherapy using nanotechnology-based carriers through the inhalational route of administration is worthy and embodies a novel upcoming targeted drug delivery "inhalational nanomedicine". It offers an effective and safer mean of lung cancer theranostics. To further strengthen this novel strategy, extensive studies at the preclinical level are required to develop a NP design with tumor specified targeting ability in lung after local delivery that has minimal effect on healthy lungs tissues and nonsignificant systemic/other organ toxicity. However, in our opinion, lung cancer being terminal, increase of life span by $1-2$ years would be highly beneficial. Particulate matter-associated fibrosis might be a 
result of long (10 years) exposure. Hence, the risk-benefit ratio of the nanoparticles is undisputed, if they are cancer therapeutics.

\section{Disclosure}

The authors report no conflicts of interest in this work.

\section{References}

1. Ramalingam SS, Owonikoko TK, Khuri FR. Lung cancer: new biological insights and recent therapeutic advances. CA Cancer J Clin. 2011;61(2):91-112.

2. Jemal A, Bray F, Center MM, Ferlay J, Ward E, Forman D. Global cancer statistics. CA Cancer J Clin. 2011;61(2):69-90.

3. American Lung Association. Trends in Lung Cancer Morbidity and Mortality. Epidemiology and Statistics Unit, Research and Scientific Affairs. Available from: http://www.lung.org/lung-disease/lungcancer/learning-more-about-lung-cancer/understanding-lung-cancer/. Accessed February 28, 2013.

4. Ferlay J, Soerjomataram I, Ervik M, et al. GLOBOCAN 2012 v1.0. Cancer Incidence and Mortality Worldwide: IARC Cancer Base Number 11 [Internet]. Lyon, France: International Agency for Research on Cancer; 2013. Available from: http://globocan.iarc.fr. Accessed December 13, 2013

5. Cochrane Database of systematic reviews. Chemotherapy can improve survival rates for nonsmall cell lung cancer. Available from: http:// summaries.cochrane.org/CD002139/chemotherapy-can-improvesurvival-rates-for-non-small-cell-lung-cancer. Accessed February 28, 2013.

6. Longo DL. Approach to the patient with cancer. In: Fauci AS, Braunwald E, Issel-bacher KJ, et al, editors. Harrison's Principles of Internal Medicine. 18th ed. New York, NY: McGraw Hill; 2011: 493-499.

7. Komaki R, Cox JD. Moss Radiation Oncology: Rationale, Technique, Results. St Louis, MO: Mosby-Year Book; 1994.

8. National Canscer Institute. SEER Stat Fact Sheet: Lung and Bronchus (2002-2008). Available from: http://seer.cancer.gov/statfacts/html/ lungb.html. Accessed December 19, 2012.

9. Roa WH, Azarmi S, Al-Hallak MH, et al. Inhalable nanoparticles, a non-invasive approach to treat lung cancer in a mouse model. J Control Release. 2011;150:49-55.

10. Lu J, Liong M, Zink JI, Tamanoi F. Mesoporous silica nanoparticles as a delivery system for hydrophobic anticancer drugs. Small. 2007; 3(8):1341-1346.

11. Kumar A, Sahoo SK, Padhee K, et al. Review on solubility enhancement techniques for hydrophobic drugs. Int J Comprehensive Pharmacy. 2011;3(3):1-7.

12. Tseng CL, Wu SY, Wang WH, et al. Targeting efficiency and biodistribution of biotinylated-EGF-conjugated gelatin nanoparticles administered via aerosol delivery in nude mice with lung cancer. Biomaterials. 2008;29:3014-3022.

13. Sung JC, Pulliam BL, Edwards DA. Nanoparticles for drug delivery to the lungs. Trends Biotechnol. 2007;25:563-570.

14. Azarmi S, Roa WH, Lobenberg R. Targeted delivery of nanoparticles for the treatment of lung diseases. Adv Drug Deliv Rev. 2008;60:863-875.

15. Akhter S, Amin S, Ahmad J, et al. Nanotechnology to combat multidrug resistance in cancer. Resistance to Targeted ABC Transporters in Cancer. Switzerland: Springer International Publishing; 2015:245-272.

16. Akhter S,Ahmad I,Ahmad MZ, etal. Nanomedicines as cancer therapeutics: current status. Curr Cancer Drug Targets. 2013;13(4):362-378.

17. Ramesh R. Nanoparticle-mediated gene delivery to the lung. Methods Mol Biol. 2008;434:301-331.

18. Yuan D, Lv Y, Yao Y, et al. Efficacy and safety of Abraxane in treatment of progressive and recurrent non-small cell lung cancer patients: a retrospective clinical study. Thorac Cancer. 2012;3(4):341-347.
19. Pilcer G, Amighi K. Formulation strategy and use of excipients in pulmonary drug delivery. Int J Pharm. 2010;392:1-19.

20. Zhang LJ, Xing B, Wu J, Xu B, Fang XL. Biodistribution in mice and severity of damage in rat lungs following pulmonary delivery of 9-nitrocamptothecin liposomes. Pulm Pharmacol Ther. 2008;21: 239-246.

21. Mansour HM, Rhee YS, Wu X. Nanomedicine in pulmonary delivery. Int J Nanomedicine. 2009;4:299-319.

22. Alipour S, Montaseri H, Tafaghodi M. Preparation and characterization of biodegradable paclitaxel loaded alginate microparticles for pulmonary delivery. Colloids Surf B Biointerfaces. 2010;81:521-529.

23. Maeda $H$. The enhanced permeability and retention (EPR) effect in tumor vasculature: the key role of tumor-selective macromolecular drug targeting. Adv Enzyme Regul. 2001;41:189-207.

24. Schreier H, Gagne L, Bock T, et al. Physicochemical properties and in vitro toxicity of cationic liposome cDNA complexes. Pharm Acta Helv. 1997;72(4):215-223.

25. Kataoka K, Matsumoto T, Yokoyama M, et al. Doxorubicin-loaded poly(ethylene glycol)-poly(beta-benzyl-1-aspartate) copolymer micelles: their pharmaceutical characteristics and biological significance. J Control Release. 2000;64:143-153.

26. Kwon G, Naito M, Yokoyama M, Okano T, Sakurai Y, Kataoka K. Block copolymer micelles for drug delivery: loading and release of doxorubicin. J Control Release. 1997;48:195-201.

27. Gill KK, Nazzal S, Kaddoumi A. Paclitaxel loaded PEG5000-DSPE micelles as pulmonary delivery platform: formulation characterization, tissue distribution, plasma pharmacokinetics, and toxicological evaluation. Eur J Pharm Biopharm. 2011;79:276-284.

28. Kalantarian P, Najafabadi AR, Haririan I, et al. Preparation of 5 -fluorouracil nanoparticles by supercritical antisolvents for pulmonary delivery. Int J Nanomedicine. 2010;5:763-770.

29. Tomoda K, Ohkoshia T, Hirotaa K, et al. Preparation and properties of inhalable nanocomposite particles for treatment of lung cancer. Colloids Surf B Biointerfaces. 2009;71:177-182.

30. Godugu C, Patel AR, Doddapaneni R, Marepally S, Jackson T, Singh M. Inhalation delivery of Telmisartan enhances intratumoral distribution of nanoparticles in lung cancer models. J Control Release. 2013;172: $86-95$.

31. Choi SH, Byeon HJ, Choi JS, et al. Inhalable self-assembled albumin nanoparticles for treating drug-resistant lung cancer. $J$ Control Release. 2015;197:199-207.

32. Azarmi S, Tao X, Chen H, et al. Formulation and cytotoxicity of doxorubicin nanoparticles carried by dry powder aerosol particles. Int J Pharm. 2006;319:155-161.

33. Xie Y, Aillon KL, Cai S, et al. Pulmonary delivery of cisplatinhyaluronan conjugates via endotracheal instillation for the treatment of lung cancer. Int J Pharm. 2010;392:156-163.

34. Subedi RK, Kang KW, Choi HK, et al. Preparation and characterization of solid lipid nanoparticles loaded with doxorubicin. Eur J Pharm Sci. 2009;37:508-513.

35. Videira M, Almeida AJ, Fabra A. Preclinical evaluation of a pulmonary delivered paclitaxel loaded lipid nanocarrier antitumor effect. Nanomedicine. 2012;8:1208-1215.

36. Videira MA, Botelho MF, Santos AC, Gouveia LF, DeLima JJ, Almeida AJ. Lymphatic uptake of pulmonary delivered radiolabelled solid lipid nanoparticles. J Drug Target. 2002;10:607-613.

37. Hu LD, Jia Y, Ding W. Preparation and characterization of solid lipid nanoparticles loaded with epirubicin for pulmonary delivery. Pharmazie. 2010;65:585-587.

38. Jyoti K, Kaur K, Pandey RS, Jain UK, Chandra R, Madan J. Inhalable nanostructured lipid particles of 9-bromo-noscapine, a tubulin-binding cytotoxic agent: in vitro and in vivo studies. J Colloid Interface Sci. 2015;445:219-230.

39. Taratula O, Kuzmov A, Shah M, Garbuzenko OB, Minko T. Nanostructured lipid carriers as multifunctional nanomedicine platform for pulmonary co-delivery of anticancer drugs and siRNA. J Control Release. 2013;171(3):349-357. 
40. Patlolla RR, Chougule M, Patel AR, Jackson T, Tata PN, Singh M. Formulation, characterization and pulmonary deposition of nebulized celecoxib encapsulated nanostructured lipid carriers. J Control Release. 2010;144:233-241.

41. Khan OF, Zaia EW, Jhunjhunwala S, et al. Dendrimer-inspired nanomaterials for the in vivo delivery of siRNA to lung vasculature. Nano Lett. 2015;15(5):3008-3016.

42. Kaminskas LM, McLeod VM, Ryan GM, et al. Pulmonary administration of a doxorubicin-conjugated dendrimer enhances drug exposure to lung metastases and improves cancer therapy. $J$ Control Release. 2014;183:18-26.

43. Pankhurst QA, Connolly J, Jones SK, et al. Applications of magnetic nanoparticles in biomedicine. J Phys D: Appl Phys. 2003;36:R167.

44. Akhter S, Ahmad Z, Singh M, et al. Cancer targeted metallic nanoparticle: targeting overview, recent advancement and toxicity concern. Curr Pharm Des. 2011;17:1834-1850.

45. Verma NK, Staunton KC, Satti A, et al. Magnetic core-shell nanoparticles for drug delivery by nebulization. J Nanobiotechnology. 2013;11:1.

46. Heyder J. Deposition of inhaled particles in the human respiratory tract and consequences for regional targeting in respiratory drug delivery. Proc Am Thorac Soc. 2004;1:315-320.

47. Plank C, Scherer F, Rudolph C. Localized nucleic acid delivery: a discussion of selected methods. In: Schleef M, editor. DNA Pharmaceuticals. Weinheim, Germany: Wiley-VCH Verlag GmbH \& Co KGaA; 2005:55-116.

48. Patton JS, Fishburn CS, Weers JG. The lungs as a portal of entry for systemic drug delivery. Proc Am Thorac Soc. 2004;1(4):338-344.

49. Taratula $\mathrm{O}$, Garbuzenko OB, Chen AM, Minko T. Innovative strategy for treatment of lung cancer: targeted nanotechnology-based inhalation co-delivery of anticancer drugs and siRNA. J Drug Target. 2011;19(10):900-914

50. Okamoto H, Shiraki K, Yasuda R, Danjo K, Watanabe Y. Chitosaninterferon- $\beta$ gene complex powder for inhalation treatment of lung metastasis in mice. J Control Release. 2011;150:187-195.

51. Tseng CL, Wang TW, Dong GC, et al. Development of gelatin nanoparticles with biotinylated EGF conjugation for lung cancer targeting. Biomaterials. 2007;28:3996-4005.

52. Tseng CL, Su WY, Yen KC, Yang KC, Lin FH. The use of biotinylated-EGF-modified gelatin nanoparticle carrier to enhance cisplatin accumulation in cancerous lungs via inhalation. Biomaterials. 2009;30:3476-3485.

53. Dames P, Gleich B, Flemmer A, et al. Targeted delivery of magnetic aerosol droplets to the lung. Nat Nanotechnol. 2007;2(8):495-499.

54. Sadhukha T, Wiedmann TS, Panyam J. Inhalable magnetic nanoparticles for targeted hyperthermia in lung cancer therapy. Biomaterials. 2013;34(21):5163-5171.

55. Shim G, Choi HW, Lee S, et al. Enhanced intrapulmonary delivery of anticancer siRNA for lung cancer therapy using cationic ethylphosphocholinebased nanolipoplexes. Mol Ther. 2013;21(4):816-824.

56. Bermudez E, Mangum JB, Wong BA, et al. Pulmonary responses of mice, rats, and hamsters to subchronic inhalation of ultrafine titanium dioxide particles. Toxicol Sci. 2004;77(2):347-357.

57. Elder A, Gelein R, Finkelstein JN, Driscoll KE, Harkema J, Oberdorster G. Effects of subchronically inhaled carbon black in three species. I. Retention kinetics, lung inflammation, and histopathology. Toxicol Sci. 2005;88(2):614-629.

Nanotechnology, Science and Applications

\section{Publish your work in this journal}

Nanotechnology, Science and Applications is an international, peer-reviewed, open access journal that focuses on the science of nanotechnology in a wide range of industrial and academic applications. It is characterized by the rapid reporting across all sectors, including engineering, optics, bio-medicine, cosmetics, textiles, resource sustainability and science. Applied research into nano-materials,
58. Warheit DB, Webb TR, Reed KL. Pulmonary toxicity screening studies in male rats with $\mathrm{TiO}_{2}$ particulates substantially encapsulated with pyrogenically deposited, amorphous silica. Part Fibre Toxicol. 2006;3:3.

59. Warheit DB, Webb TR, Reed KL, Frerichs S, Sayes CM. Pulmonary toxicity study in rats with three forms of ultrafine- $\mathrm{TiO}_{2}$ particles: differential responses related to surface properties. Toxicology. 2007; 230(1):90-104.

60. Warheit DB, Webb TR, Sayes CM, Colvin VL, Reed KL. Pulmonary instillation studies with nanoscale $\mathrm{TiO}_{2}$ rods and dots in rats: toxicity is not dependent upon particle size and surface area. Toxicol Sci. 2006;91(1):227-236.

61. Lam CW, James JT, McCluskey R, Hunter RL. Pulmonary toxicity of single-wall carbon nanotubes in mice 7 and 90 days after intratracheal instillation. Toxicol Sci. 2004;77(1):126-134.

62. Mercer RR, Scabilloni J, Wang L, et al. Alteration of deposition pattern and pulmonary response as a result of improved dispersion of aspirated single-walled carbon nanotubes in a mouse model. Am J Physiol Lung Cell Mol Physiol. 2008;294(1):87-97.

63. Warheit DB, Laurence BR, Reed KL, Roach DH, Reynolds GA, Webb TR. Comparative pulmonary toxicity assessment of single-wall carbon nanotubes in rats. Toxicol Sci. 2004;77(1):117-125.

64. Curtis J, Greenberg M, Kester J, Phillips S, Krieger G. Nanotechnology and nanotoxicology: a primer for clinicians. Toxicol Sci. 2006;25(4): 245-260.

65. Hagens WI, Oomen AG, de Jong WH, Cassee FR, Sips AJ. What do we (need to) know about the kinetic properties of nanoparticles in the body? Regul Toxicol Pharmacol. 2007;49(3):217-229.

66. Moghimi SM, Hunter AC, Murray JC. Nanomedicine: current status and future prospects. FASEB J. 2005;19(3):311-330.

67. Oberdorster G, Oberdorster E, Oberdorster J. Nanotoxicology: an emerging discipline evolving from studies of ultrafine particles. Environ. Health Perspect. 2005;113(7):823-839.

68. Medina C, Santos-Martinez MJ, Radomski A, Corrigan OI, Radomski MW. Nanoparticles: pharmacological and toxicological significance. Br J Pharmacol. 2007;150(5):552-558.

69. Oberdorster G, Sharp Z, Atudorei V, et al. Translocation of inhaled ultrafine particles to the brain. Inhal Toxicol. 2004;16(6-7):437-445.

70. Lanone S, Boczkowski J. Biomedical applications and potential health risks of nanomaterials: molecular mechanisms. Curr Mol Med. 2006;6(6):651-663.

71. Badea I, Wettig S, Verrall R, Foldvari M. Topical non-invasive gene delivery using gemini nanoparticles in interferon-gamma-deficient mice. Eur J Pharm Biopharm. 2007;65(3):414-422.

72. Fernandez L, Sua LF, Camargo R, Basante M, Gutierrez O, Munoz J. Evaluation of the pulmonary inflammatory response in murine biomodels exposed to modified titanium oxide (tio2-Modified). Biomedical Research. 2015;1(2):3.

73. Garnett MC, Kallinteri P. Nanomedicines and nanotoxicology: some physiological principles. Occup Med (Lond). 2006;56(5):307-311.

74. Lacerda L, Bianco A, Prato M, Kostarelos K. Carbon nanotubes as nanomedicines: from toxicology to pharmacology. Adv Drug Deliv Rev. 2006;58(14):1460-1470.

75. Witzmann FA, Monteiro-Riviere NA. Multi-walled carbon nanotube exposure alters protein expression in human keratinocytes. Nanomedicine. 2006;2(3):158-168.

76. Schins RP, Knaapen AM. Genotoxicity of poorly soluble particles. Inhal Toxicol. 2007;19(1):189-198.

particles, nano-structures and fabrication, diagnostics and analytics, drug delivery and toxicology constitute the primary direction of the journal. The manuscript management system is completely online and includes a very quick and fair peer-review system, which is all easy to use. Visit http://www.dovepress.com/ testimonials.php to read real quotes from published authors. 